\title{
Anti-angiogenic therapy for normalization of tumor vasculature: A potential effect of Buyang Huanwu decoction on nude mice bearing human hepatocellular carcinoma xenografts with high metastatic potential
}

\author{
LIANG MIN ${ }^{1}$, WEI LING ${ }^{2}$, RONG HUA ${ }^{3}$, HONG $^{1}{ }^{1}$, SHENXU CHEN $^{1}$, \\ HAIQIAO WANG ${ }^{1}$, LUMEN TANG ${ }^{1}$ and WENJI SHANGGUAN ${ }^{1}$ \\ Departments of ${ }^{1}$ Traditional Chinese Medicine, ${ }^{2}$ Gastrointestinal Surgery and ${ }^{3}$ Hepatopancreatobiliary Surgery, \\ Ren Ji Hospital, School of Medicine, Shanghai Jiao Tong University, Shanghai 200127, P.R. China
}

Received March 4, 2015; Accepted December 15, 2015

DOI: $10.3892 / \mathrm{mmr} .2016 .4854$

\begin{abstract}
The present study aimed to investigate the effect of Buyang Huanwu decoction (BYHWD) on tumor growth, metastasis and angiogenesis in nude mice bearing human hepatocellular carcinoma (HCC) HCCLM3 xenografts. A total of 96 nude mice bearing HCCLM3 xenografts were randomly divided into four groups: BYHWD group (LB), Yi-qi decoction group (LY), Huo-xue decoction group (LH) and model group (LM). Each of these groups was divided into three subgroups $(\mathrm{n}=8)$, which were observed on days $21,25,38$ following treatment, respectively. The tumor weights, volumes and pulmonary metastases were recorded. The expression of CD105 and the microvessel density (MVD) were assessed, and the expression levels of vascular endothelial growth factor (VEGF), hypoxia-inducible factor $1 \alpha$ (HIF-1 $\alpha$ ), and regulator of G protein signaling 5 (RGS-5) were analyzed using immunohistochemical staining. Compared with the LM group, no significant decrease in tumor weight or volume were observed in the herbal medicine treatment groups, the number of the metastases in the lungs decreased, whereas the expression levels of RGS-5 and HIF-1 $\alpha$ decreased in the LB group on day 35. However, the expression levels of VEGF increased in the LB group on days 28 and 35 post-treatment. The results of the present study suggested that BYHWD may inhibit angiogenesis and metastasis by affecting the expression levels of VEGF, RGS-5 and HIF-1 $\alpha$, and suggested that BYHWD may
\end{abstract}

Correspondence to: Dr Liang Min, Department of Traditional Chinese Medicine, Ren Ji Hospital, School of Medicine, Shanghai Jiaotong University, 2000 Jiangyue Road, Pujiang, Minhang, Shanghai 200127, P.R. China

E-mail: liangmindr@163.com

Key words: human hepatocellular carcinoma, tumor weight, vascular endothelial growth factor, microvessel density, pulmonary metastasis contribute to the tumor microenvironment and vasculature normalization in HCC.

\section{Introduction}

Hepatocellular carcinoma (HCC), the predominant form of primary liver cancer, is one of the most frequent types of human malignancy and the third leading cause of cancer-asssociated mortality worldwide (1). It is reported that $>700,000$ cases of HCC are diagnosed annually, with a 5-year-survival rate of $\leq 10 \%$ (2). Effective treatment of HCC remains a significant challenge (3). Even in patients who have received surgical management of HCC, including resection and liver transplantation, recurrence and metastasis remain major obstacles in further prolonging the survival rates of patients with HCC (3). Therefore, understanding the pathogenesis of HCC and examining more efficient therapeutic strategies to prevent HCC metastasis and recurrence are important.

Sustained proliferation, invasion, metastasis, and angiogenesis are important features of cancer (1). Therefore, preventing the growth and metastatic spread of HCC is a principal aim of HCC therapy. Angiogenesis is known as the formation of new blood vessels from pre-existing vessels, as well as the remodeling of the newly formed vascular network (4). In addition, angiogenesis is widely considered as pivotal procedures in removing metabolic waste products, to nourish the growing tumor (4). Pang and Poon (5) revealed that $\mathrm{HCC}$ was a hypervascular carcinoma characterized by neovascularization, and vascular endothelial growth factor (VEGF), and has a significant role in the angiogenesis of HCC. Evidence has shown that angiogenesis is a critical factor for tumor growth and metastasis, and tumor growth was not considerable when emerging new capillary blood vessels were inhibited (6). Targeting angiogenesis has been validated in several types of solid tumor (7-9). For example, Zhao et al reported that inhibition of tumor angiogenesis by suppression of the Notch signaling pathway may be a potential mechanism underlying the antitumor activity of total alkaloids of Rubus alceifolius Poir in a mouse model of HCC (7). Thus, 
anti-angiogenic strategy has been shown promising effects for HCC therapy.

Oriental herbal medicine has been used since ancient times to treat malignancies (10). Hu et al indicated that Chinese herbal medicine was emerging as a treatment of choice due to its multi-target, multilevel and coordinated intervention effects against HCC (11). For example, Han et al reported that Chinese medicines can exert anti-angiogenic effects through regulating the expression of VEGF or reducing the activities of angiogenic factor receptors, or by inhibiting the proliferation of endothelial cells (12). In addition, blood-activating and stasis-eliminating herbs, including Salvia miltiorrhiza and Turmeric rhizome attenuated tumor angiogenic activities (12). Dong et al revealed that Cucurbitacin E, which is extracted from Chinese medicine, inhibited tumor angiogenesis through a specific pathway (13). In addition, Huang et al reported that the herbal compound extract 'Songyou Yin' inhibited the growth and invasion of HCC, and decreased microvessel density and the abundance of VEGF (14). Buyang Huanwu decotion (BYHWD), a traditional Chinese medicine functionally characterized by activated energy, $Q i$, invigorates the body, enhances blood circulation and meridian circulation, and has been traditionally used in the treatment of stroke and paralysis for centuries (15). Cai et al found that BYHWD improved the recovery of neurological function, stimulated neural proliferation, reduced infarction volume and modulated the expression of VEGF and its receptor fetal liver kinase in transient focal cerebral ischemic rat brains (16). In addition, Jain et al demonstrated that certain anti-angiogenic agents transiently normalized the abnormal vasculature structure and function, improving its efficiency for drug delivery and alleviating hypoxia (17). Additionally, several traditional Chinese herbal drugs have exhibited anti-angiogenic effects and enhanced normalization of tumor vasculature (18). However, the detail and potential mechanism underlying the effects of BYHWD on HCC remains to be elucidated.

The established metastatic model of human HCC in nude mice exhibits the metastatic ability, transplantability and manifestations reminiscent of tumor behavior in patients with HCC (19). The present study aimed to investigate the effect and the potential mechanism of BYHWD on tumor growth, metastasis and angiogenesis in nude mice bearing human HCC HCCLM3 xenografts.

\section{Materials and methods}

Characterization and preparation of herbal medicine. The uses of the Chinese medicine formula, BYHWD, and its disassembled prescriptions, Yiqi decoction (YQD) and Huoxue decoction (HXD), in the present study were authorized according to the medical publications; Formulas of Chinese medicine (20) and the Chinese Pharmacopoeia (21). BYHWD was composed of seven medicinal components: $120 \mathrm{~g}$ milkvetch root, $6 \mathrm{~g}$ Chinese angelica, $5 \mathrm{~g}$ red peony root, $3 \mathrm{~g}$ earth worm, $3 \mathrm{~g}$ Szechwan lovage rhizome, $3 \mathrm{~g}$ peach seed and $3 \mathrm{~g}$ safflower. YQD consisted of only $120 \mathrm{~g}$ milkvetch root, and HXD consisted of the remaining medicinal components: $6 \mathrm{~g}$ Chinese angelica, $5 \mathrm{~g}$ red peony root, $3 \mathrm{~g}$ earth worm, $3 \mathrm{~g}$ Szechwan lovage rhizome, $3 \mathrm{~g}$ peach seed and $3 \mathrm{~g}$ safflower. All the herbal components were purchased from Shanghai
Pharmacy (Shanghai, China), and authenticated by experts at the Department of Pharmacology, Shanghai University of Chinese Medicine (Shanghai, China). The three medicinal herb decoctions were prepared as follows. The mixture of the crude drugs were soaked in $500 \mathrm{ml}$ distilled water and decocted by boiling for $35 \mathrm{~min}$. The resulting decoction was then filtered through a $0.22 \mu \mathrm{m}$ polytetrafluoroethylene filter (Whatman; GE Healthcare Life Sciences, Chalfont, UK) and collected. The remnants were added to $350 \mathrm{ml}$ distilled water, and decocted by boiling was continued for $20 \mathrm{~min}$, followed by filtering. The filtered decoctions were combined and condensed to a specific dose. The doses were calculated according to the formula: $\mathrm{dB}=\mathrm{dA}{ }^{*} \mathrm{RB} / \mathrm{RA}^{*}(\mathrm{WA} / \mathrm{WB})^{1 / 3}$ (22), where $\mathrm{dA}$ represents the human dose and $\mathrm{dB}$ represents the mouse dose; WA is the average human weight $(60 \mathrm{~kg})$ and $\mathrm{WB}$ is the average mouse weight $(0.2 \mathrm{~kg})$. $R$ is the build coefficient $(R A=1.00 ; R B=0.59)$. The combined filtrates of BYHWD, YQD and HXD were condensed to $1.03,0.56$ and $0.166 \mathrm{~g} / \mathrm{ml}$, respectively. All the combined filtrates were stored at $4^{\circ} \mathrm{C}$ until use.

Animals and metastatic model of human HCC in nude mice. Male and female athymic BALB/c nu/nu mice (15-50 g; 4-6 weeks old) were purchased from Shanghai Slack Experimental Animals Co., Ltd. (Shanghai, China) and maintained under specific pathogen-free conditions. All mice were handled according to the Guidance Suggestions for the National Institutes for the Care and Use of Laboratory Animals (23). The study was approved by the ethics committee of Shanghai University of Traditional Chinese Medicine.

The HCCLM3 HCC cell line, which was cultured in Dulbecco's modified Eagle's medium supplemented with $10 \%$ fetal bovine serum (Gibco; Thermo Fisher Scientific, Inc., Waltham, MA, USA), was established at the Liver Cancer Institute of Fudan University (Fudan, China) (24). The human HCC tumor models were established using HCCLM3 in nude mice via the orthotopic implantation of intact metastatic tumor tissue, as described in previous reports $(25,26)$. Briefly, following the acquirement of HCCLM3, $5 \times 10^{6}(0.2 \mathrm{ml})$ cells were injected subcutaneously into four nude mice (male or female). When the subcutaneous tumor had reached $\sim 1.5 \mathrm{~cm}$ in diameter, the mice were sacrificed by cervical dislocation. The tumor tissue was removed, cut into small sections $\left(\sim 1 \mathrm{~mm}^{3}\right)$ and implanted into the liver of separate recipient mice, which were kept in standard facilities. This animal model showed $100 \%$ spread into the liver and metastasis to the lungs. Abnormal serum $\alpha$-fetoprotein was excreted, and hepatitis B surface antigens were also identified in this model.

Mice grouping and treatment. A total of 96 nude mice bearing orthotopic xenografts were randomly divided into four groups: Model control group (LM); BYHWD-treated group (LB), YQD-treated group (LY) and HXD-treated group (LH). Each of these groups was randomized into three subgroups, which were treated consecutively for 21 days (LM21, LB21, LY21 and LH21), 28 days (LM28, LB28, LY28 and LH28), and 35 days (LM35, LB35, LY35, and LH35), respectively. Each subgroup contained eight mice. Treatment began the day following that on which xenograft surgery was performed. The groups of animals $(n=8)$ were orally gavaged with $0.2 \mathrm{ml}$ of the respective treatment solution twice daily, with the exception 
of the animals in the model group, which received $0.2 \mathrm{ml}$ of $0.9 \%$ sodium chloride solution twice daily. Daily general observations and weekly mice body weights were all recorded.

Parameters observed and grading of lung metastasis. The mice were sacrificed after 21,28 and 35 days, respectively. The tumors were removed, images were captured, and the tumor tissues were weighed and processed for histology. Following autopsy, the longest (a) and the smallest (b) diameters of the tumors were measured using a slide gauge (Control Co., Friendswood, TX, USA) under an operating microscope (OPMI Pentero; Carl Zeiss, Oberkochem, Germany). The tumor volume was calculated as follows: Tumor volume $=a b^{2} / 2$. Both lungs of each mouse were removed, and paraffin blocks of $10 \%$ buffered formalin (Tissue Prep-II; Thermo Fisher Scientific, Inc.)-fixed samples of the lungs were prepared. A total of five coronal sections were selected from each paraffinized lung sample, with each coronal section cut consecutively into five slices. Serial sections were cut at $4 \mu \mathrm{m}$, and 20 slices in the group were randomly selected and stained with hematoxylin and eosin (Beijing Solarbio Science \& Technology Co., Ltd., Beijing, China) to determine the presence of lung metastases under a light microscope (Leica DMIRBI; Leica Microsystems $\mathrm{GmbH}$, Wetlzar, Germany). If at least one in these 20 slices was found to exhibit lung metastasis, the mice were confirmed to have lung metastasis. The degree of lung metastasis was graded by the number $(\mathrm{N})$ of tumor cells counted in the maximum section of a solitary pulmonary metastatic nodule: Grade I, $\mathrm{N}<20$; grade II, $\mathrm{N}=20-50$; grade III, $\mathrm{N}=50-100$; grade IV, $\mathrm{N}>100$.

Immunohistochemical assessment of microvessel density. Paraffin-embedded tumor tissues were cut into 4- $\mu \mathrm{m}$-thick sections, dewaxed in xylene (Sinopharm Chemical Reagent Co., Ltd., Shanghai, China) and dehydrated in ethanol. Two-step methods (EnVision ${ }^{\mathrm{TM}}$ system; DakoCytomation, Glostrup, Denmark) were used for CD105 staining. Briefly, the endogenous peroxidase activity was quenched with $3 \%$ $\mathrm{H}_{2} \mathrm{O}_{2}$ (Sinopharm Chemical Reagent Co., Ltd.) in methanol for 10 min. The CD105 antigen was unmasked by microwave oven (Panasonic 1380W; Panasonic Corporation, Osaka, Japan) pre-treatment in $\mathrm{pH} 9.0$ ethylene diamine tetraacetic acid buffer (Invitrogen; Thermo Fisher Scientific, Inc.), and then cooled to room temperature. The sections were then incubated with the following primary monoclonal antibodies: mouse anti-endoglin anti-CD105 (SN6 h; DAKO; 1:50 dilution) for $2 \mathrm{~h}$ at $37^{\circ} \mathrm{C}$, followed by incubation with rabbit anti-mouse horseradish peroxidase (HRP)-conjugated streptavidin (P0397; DAKO; 1:50 dilution) for $45 \mathrm{~min}$ at $37^{\circ} \mathrm{C}$. Antibody binding was visualized with 3,3-diaminobenzidine (DAB; Sigma-Aldrich, St. Louis, MO, USA), and the sections were counterstained with Mayer's hematoxylin (Sgma-Aldrich). Prior to each subsequent step, the sections were washed extensively with phosphate-buffered saline (PBS) twice for $5 \mathrm{~min}$. Tissues incubated with PBS as the primary antibody served as negative controls.

The quantification of MVD was performed in accordance with a previously described method (27). Briefly, the three most vascularized areas of the tumor tissues were initially identified under a low-power field $(\mathrm{x} 40)$. The numbers of microvessels were then counted in each of these areas under a high-power field (x400) using a LEICA DMLB light microscope (Leica Microsystems $\mathrm{GmbH}$ ). Any brown-stained single endothelial cells, or cell clusters with or without a discernible lumen and separated from adjacent microvessels and other connective tissue elements, were considered to be an individual, countable microvessel. The mean value of three $\mathrm{x} 400$ field $\left(0.40 \mathrm{~mm}^{2}\right)$ counts was recorded as the MVD of the section. All microvessel counts were performed independently by two investigators, who were blinded to the clinicopathological data. The rate of disagreement between the two investigators' analyses was $<10 \%$.

Immunohistochemical (IHC) detection of the expression levels of VEGF, regulator of $G$ protein signaling $5(R G S-5)$ and hypoxia-inducible factor $1 \alpha(H I F-1 \alpha)$. Immunohistochemistry was performed using an $\mathrm{EnVision}^{\mathrm{TM}}$ method using the reagents supplied within the kit. Briefly, $4 \mu \mathrm{m}$-thick sections were cut consecutively from paraffin-embedded tumor tissue. Following deparaffinization and dehydration, the tissue sections were repaired for $40 \mathrm{~min}$ with $10 \%$ ethylenediamine tetraacetic acid (Invitrogen; Thermo Fisher Scientific, Inc.). The slides were then incubated with either primary VEGF monoclonal mouse antibody (clone VG1; M7273; DAKO; 1:100 dilution) for $60 \mathrm{~min}$ at $37^{\circ} \mathrm{C}$, primary rabbit ant-mouse RGS-5 polyclonal antibody (SAB1411523; Sigma-Aldrich; 1:80 dilution) overnight at $4^{\circ} \mathrm{C}$, or primary rabbit anti-mouse HIF-1 $\alpha$ polyclonal antibody (sc-10790; Santa Cruz Biotechnology, Inc., Dallas, TX, USA; 1:200 dilution) overnight at $4^{\circ} \mathrm{C}$. Following three rinses in PBS, the slides were incubated with the secondary antibodies (goat anti-mouse/rabbit unbiotinylated antibody-HRP; EnVision ${ }^{\mathrm{TM}}$ System; K5007; DAKO, 1:100 dilution) for $30 \mathrm{~min}$ at $37^{\circ} \mathrm{C}$. The tissue staining was visualized with DAB substrate (DAKO), and the sections were counterstained with Mayer's hematoxylin. Antibody binding was visualized with DAB. Prior to each subsequent-step, the sections were washed extensively with $0.01 \mathrm{M}$ (pH 7.4) triethanolamine-buffered saline (TBS; Wuhan Boster Biological Technology, Ltd., Wuhan, China). Tissues incubated with TBS as the primary antibody served as negative controls.

Positive staining was located in the cytoplasm for VEGF and RGS-5. Positive HIF-1 $\alpha$ expression was located as brown staining, predominantly in the nucleus. A total of five areas were counted under $\mathrm{x} 400$ field. The levels of expression were graded, as follows: Negative (-), $<5 \%$ of the cancerous cells were positively stained. Weak positive (+), $5-25 \%$ of the cancerous cells were positively stained and the cytoplasm was light brown. Positive (++), 25-50\% of the cancer cells were positively stained, and positive cells were light brown with a granule-like appearance in the cytoplasm. Strongly positive $(+++), 50 \%$ of the cancer cells were positively stained and the cytoplasm was dark brown. The level of expression was represented by the grade: $\geq 5 \%=$ positive, $<50 \%=$ low level expression, $\geq 50 \%$ high level expression. The percentage of positive tumor cells and the mean optical density (OD) values were calculated.

Statistical analysis. The data are presented as the mean \pm standard deviation, and were analyzed using SPSS 17.0 software (SPSS, Inc., Chicago, IL, USA). One-way analysis of variance 


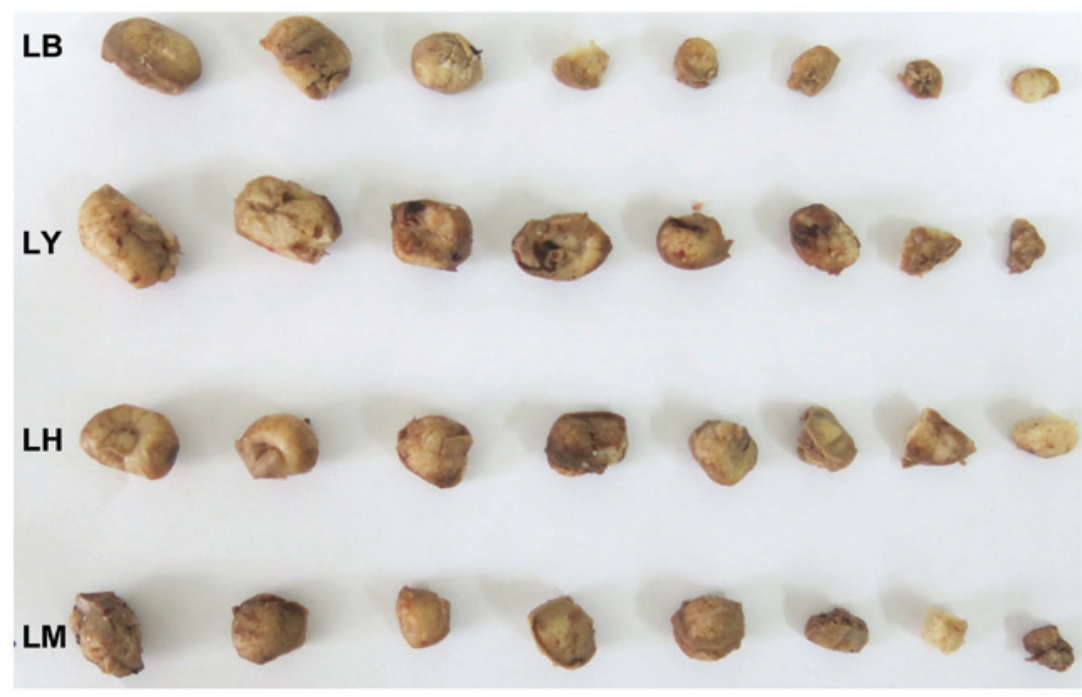

Figure 1. Tumors from nude mice 35 day following herbal medicine treatment. P>0.05, tumor size between groups. LM, model control; LB, Buyang Huanwu decotion-treated; LY, Yiqi decoction-treated; LH, Huoxue decoction-treated.

A

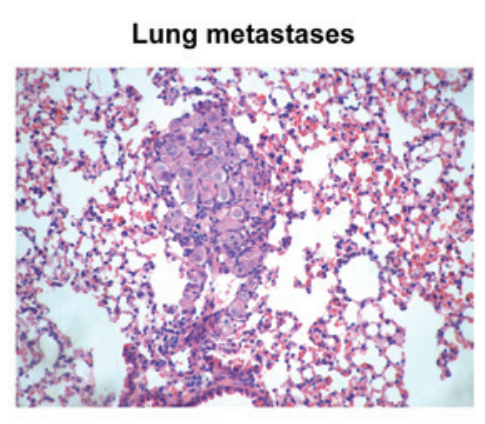

B
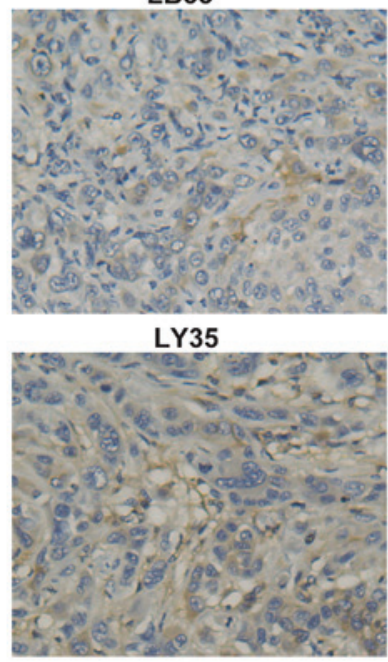

LH35

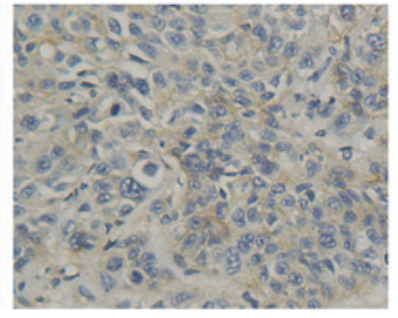

LM35

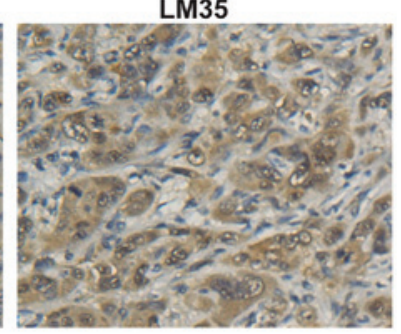

Figure 2. Lung metastases and IHC determination of the expression of CD105. (A) Metastases of HCC in the lung (hematoxylin and eosin staining; original magnification, x200). (B) IHC was used to evaluate the expression levels of CD105 in HCC tissues from nude mice of different groups 35 day following herbal medicine treatment (3,3-diaminobenzidine staining; original magnification, x400). HCC, hepatocellular carcinoma; IHC, immunohistochemistry; LM, model control; LB, Buyang Huanwu decotion-treated; LY, Yiqi decoction-treated; LH, Huoxue decoction-treated.

and Student's $t$-test were used for comparisons between groups. $\mathrm{P}<0.05$ was considered to indicate a statistically significant difference.

\section{Results}

BYHWD treatment has no significant effect on tumor growth At the point at which the mice were sacrificed, lumps were grossly visible. The tumors removed from the mice in the treatment groups and model control group at day 35 are shown in Fig. 1. The tumor weights and volumes in each group are presented in Table I. The results revealed that the changes in tumor weight $(\mathrm{g})$ and tumor volume $\left(\mathrm{mm}^{3}\right)$ in the LB35 group were smaller, compared with those in the LM35 group, but without statistical significance $(\mathrm{P}>0.05$; Table I). No animals experienced weight loss of $>10 \%$.
BYHWD treatment decreases the number of lung metastases in $H C C$. Visible metastases were observed in all groups, as shown in Fig. 2A, the number of which were recorded. At 35 days post-treatment, the rate of metastasis to the lungs was $100 \%$ in all groups, however, the number of metastases in the lungs of the treatment groups were significantly lower, compared with the number in the LM group $(\mathrm{P}<0.05)$. In the LB group, the number of lung metastases was the lowest $(\mathrm{P}<0.05)$. The majority of the lung sections in the LM35 group were observed to contain scattered hemorrhagic spots. The degree of lung metastasis ranged between grade I and grade III, with $10 \%$ of metastases in the LB35 group being grade II and the others in the group being grade I. In the LH35 group, $40 \%$ were grade I and 60\% were grade III. In the LY35 group, $20 \%$ were grade III and the others in the group were grade I. 
Table I. Comparison of tumor growth and metastases between groups 35 days following treatment.

\begin{tabular}{lccc}
\hline Group & $\mathrm{n}$ & $\begin{array}{c}\text { Tumor weight } \\
(\mathrm{g})\end{array}$ & $\begin{array}{c}\text { Tumor volume } \\
\left(\mathrm{mm}^{3}\right)\end{array}$ \\
\hline LB & 8 & $0.55 \pm 0.50$ & $440.70 \pm 401.15$ \\
LY & 8 & $1.08 \pm 0.70$ & $765.33 \pm 534.96$ \\
LH & 8 & $0.90 \pm 0.45$ & $718.41 \pm 376.01$ \\
LM & 8 & $0.62 \pm 0.34$ & $533.47 \pm 336.82$ \\
\hline
\end{tabular}

Values are expressed as the mean \pm standard deviation. $\mathrm{P}>0.05$, compared with the LM group. LM, model control; LB, Buyang Huanwu decotion-treated; LY, Yiqi decoction-treated; LH, Huoxue decoction-treated.

BYHWD treatment decreases the expression of CD105 and $M V D$ counts. A significant reduction in the number of stained regions were revealed following herbal medicine treatment. The expression levels of CD105 in the herbal medicine treatment groups were weak or even negative, whereas the expression of CD105 was more marked in the control group (Fig. 2B). Only the results at 35 day post-treatment have been presented, as the results on days 21 and 28 were similar to those at day 35 . The newborn endothelial cells were stained brown or yellow, and were sinusoidally distributed in the capillary walls of the fiber interval and portal area of the liver tissues (Fig. 2B). In the 35 day groups, microvessel counting revealed that the MVD counts in the LB, LY, LH and LM groups at a high-power field (x400) were 5.60 $\pm 3.02,6.08 \pm 1.42,8.07 \pm 2.65$ and $12.00 \pm 4.45$, respectively. The MVD in the LM group was higher, compared with those in the medicine treatment groups, and the lowest MVD was found in the LB35 group $(\mathrm{P}<0.05)$. These results showed that BYHWD treatment caused a marginal decrease in MVD.

BYHWD affects the expression levels of VEGF, RGS-5 and $H I F-1 \alpha$. To further determine whether BYHWD treatment inhibits the angiogenesis of HCC by inhibiting VEGF or other factors, the present study measured the expression levels of VEGF, RGS-5 and HIF-1 $\alpha$. The expression values of VEGF (positive cell rate $\mathrm{x}$ OD) are shown in Fig. 3B. The expression values in the LB35, LY35, LH35 and LM35 groups were $285.16 \pm 75.80,228.12 \pm 81.45,231.12 \pm 81.84$ and $200.00 \pm 80.96$, respectively. The results showed that the expression of VEGF was higher in the LB35 group, compared with LM35 group $(\mathrm{P}<0.05)$, and the IHC results revealed a significant increase in the expression levels of VEGF in the herbal medicine treatment groups, compared with LM group (Fig. 3A). The expression levels of VEGF were also significantly increased in the LB28 group, compared with the LM28 group $(\mathrm{P}<0.05$; data not shown).

The IHC results and the expression values of RGS-5 are presented in Fig. 4 and Table II. Statistical analysis indicated that the expression levels of RGS-5 in herbal medicine treatment groups were significantly different from that in the model control group. The results showed that the expression value of RGS-5 on day 28 following treatment in the LB, LY, LH and
A
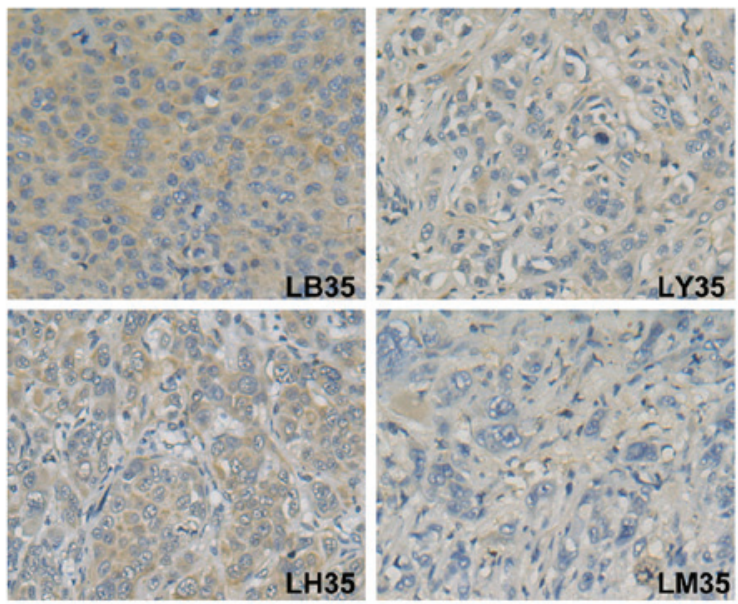

B

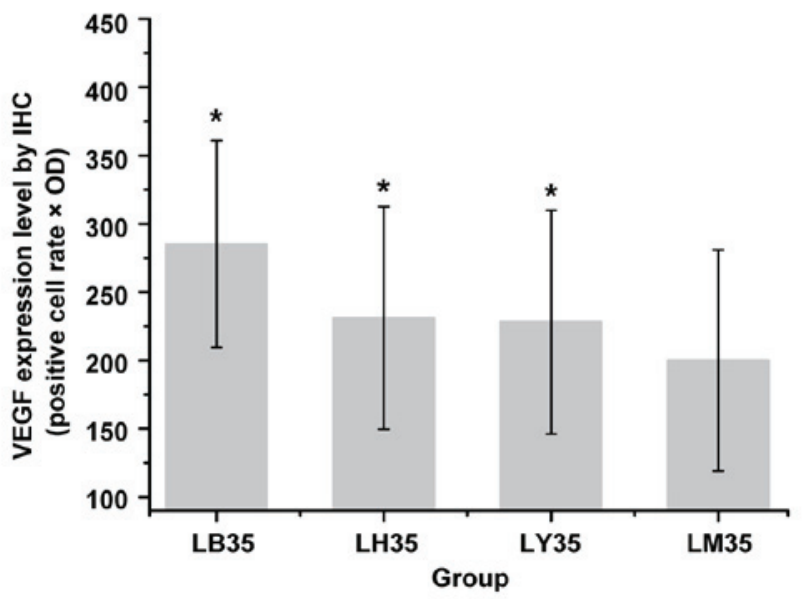

Figure 3. Expression levels of VEGF in HCC tissues from nude mice of different groups 35 days following treatment (3,3-diaminobenzidine staining; original magnification, $x 400$ ). (A) Quantification of IHC results of the expression of VEGF. (B) Expression levels were determined as the positive cell rate $\mathrm{x}$ OD. data Data are presented as the mean \pm standard deviation. ${ }^{*} \mathrm{P}<0.05$, compared with the LM35 group. HCC, hepatocellular carcinoma; LM, model control; LB, Buyang Huanwu decotion-treated; LY, Yiqi decoction-treated; LH, Huoxue decoction-treated; IHC, immunohistochemistry; VEGF, vascular endothelial growth factor; OD, optical density.

LM groups were $262.64 \pm 36.33,280.75 \pm 32.46,281.00 \pm 31.64$ and $312.87 \pm 39.36$, respectively. The expression values of RGS-5 in the LB35, LY35, LH35 and LM35 groups were $294.16 \pm 63.70,303.66 \pm 30.02,310.20 \pm 51.23$ and $349.25 \pm 48.27$, respectively. These results showed that the herbal medicine treatment groups had lower expression levels of RGS-5 on days 28 and 35, compared with the corresponding time points in the LM group $(\mathrm{P}<0.05)$. BYHWD treatment exerted a more significant inhibitory effect, compared with the other herbal medicines. However, no significant difference was found on day 21 in the medicine treatment groups, compared with the model group ( $\mathrm{P}>0.05$; Fig. 4; Table II).

The present study also examined the change in expression of HIF-1 $\alpha$ following herbal medicine treatment, compared with the model control group (Fig. 5). The result showed that the expression of HIF-1 $\alpha$ in the tumor tissue was downregulated by BYHWD and HXD treatment, compared with the LM group, at day 35 post-treatment $(\mathrm{P}<0.05)$. The expression values (positive cell rate $\mathrm{x}$ OD) are shown in Table III. 

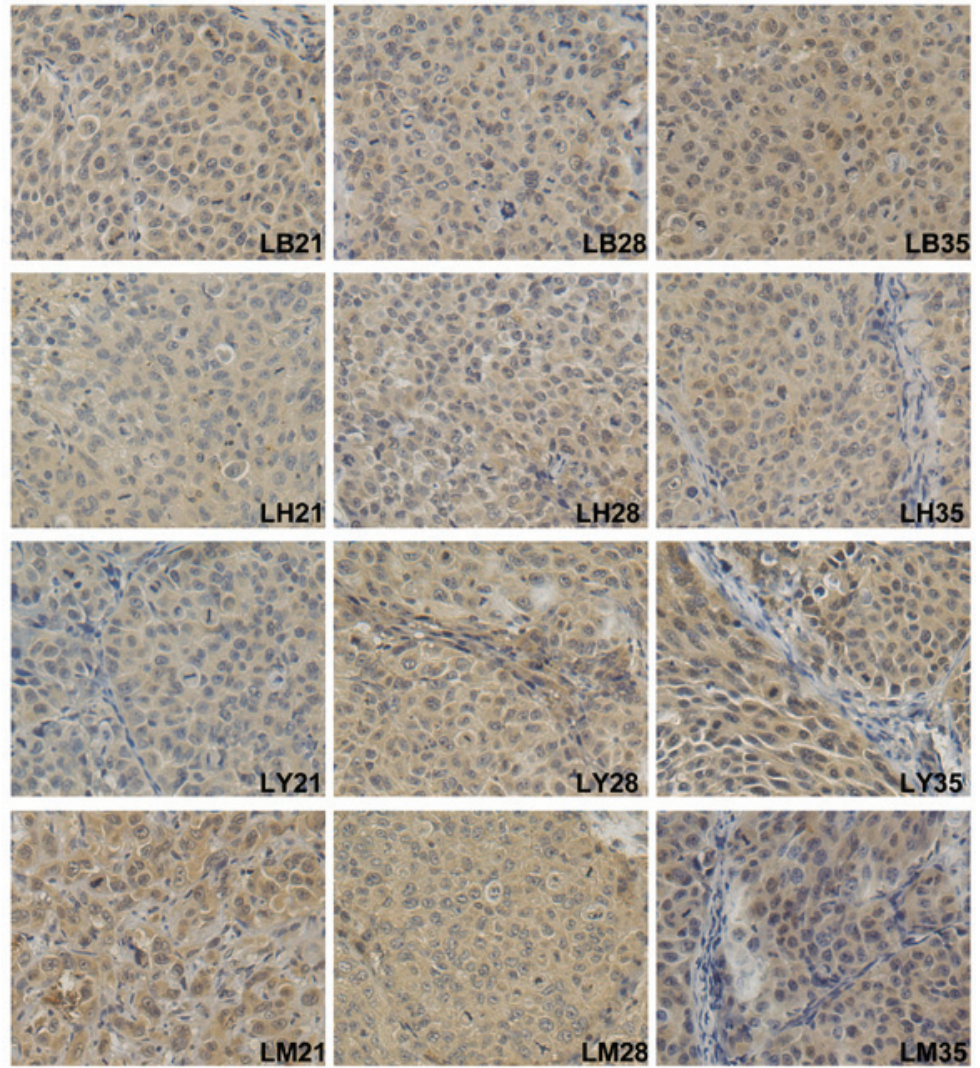

Figure 4. Representative immunostaining for RGS-5 in hepatocellular carcinoma tissues from nude mice in different treatment groups on days 21,28 and 35 following treatment (3,3-diaminobenzidine staining; original magnification, x400). LM, model control; LB, Buyang Huanwu decotion-treated; LY, Yiqi decoction-treated; LH, Huoxue decoction-treated.
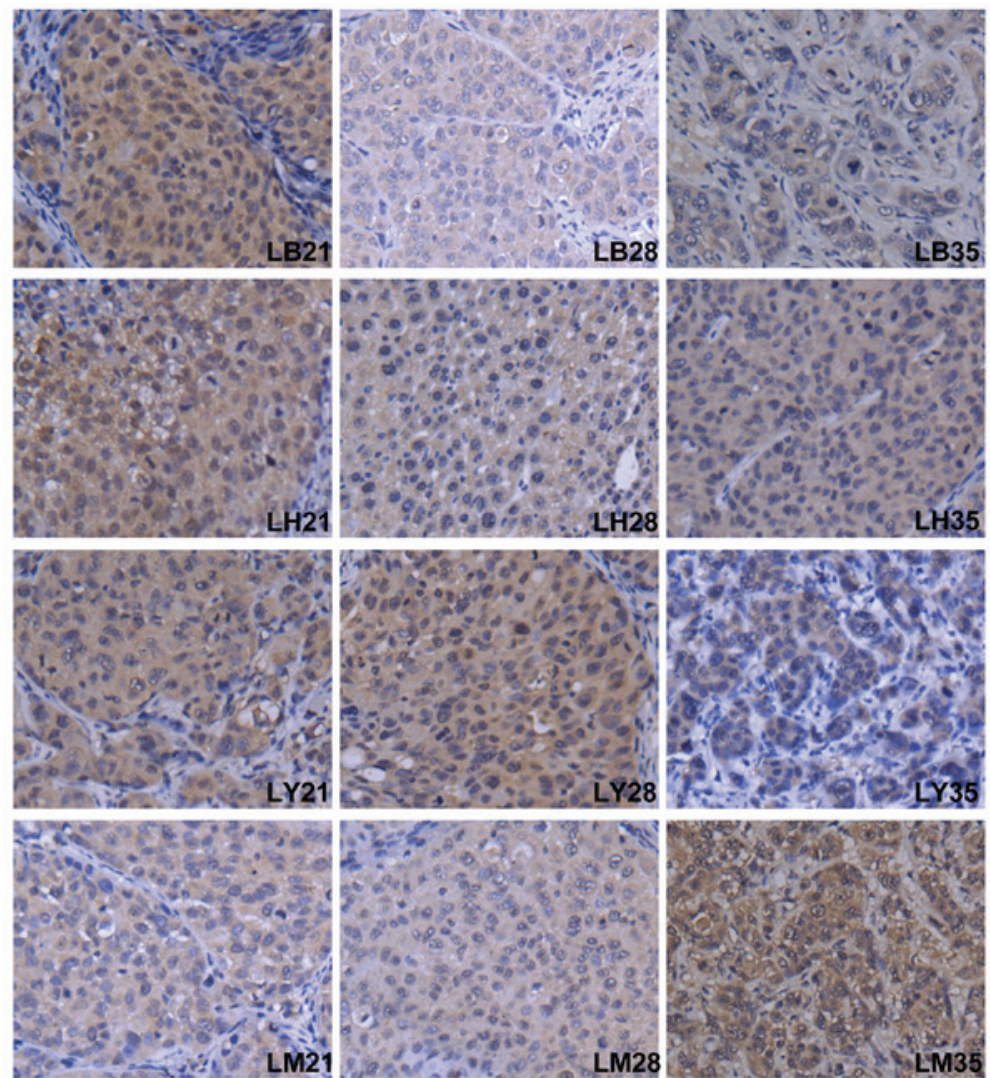

Figure 5. Immunohistochemical staining of the expression of HIF-1 $\alpha$ in hepatocellular carcinoma tissues from nude mice of different groups on different days (3,3-diaminobenzidine staining; original magnification, x400). LM, model control; LB, Buyang Huanwu decotion-treated; LY, Yiqi decoction-treated; LH, Huoxue decoction-treated. 
Table II. Comparison of expression levels of regulator of $\mathrm{G}$ protein signaling 5 .

\begin{tabular}{lccc}
\hline Group & Day 21 & Day 28 & Day 35 \\
\hline LB & $241.66 \pm 76.90$ & $262.64 \pm 36.33^{\mathrm{a}}$ & $294.16 \pm 63.70^{\mathrm{b}}$ \\
LY & $271.16 \pm 46.44$ & $280.75 \pm 32.46^{\mathrm{a}}$ & $303.66 \pm 30.02^{\mathrm{b}}$ \\
LH & $268.12 \pm 52.34$ & $281.00 \pm 31.64^{\mathrm{a}}$ & $310.20 \pm 51.23^{\mathrm{b}}$ \\
LM & $285.27 \pm 49.69$ & $312.87 \pm 39.36$ & $349.25 \pm 48.27$ \\
\hline
\end{tabular}

${ }^{\mathrm{a}} \mathrm{P}<0.05$, compared with the $\mathrm{LM} 28$ group. ${ }^{\mathrm{b}} \mathrm{P}<0.05$, compared with the LM35 group. LM, model control; LB, Buyang Huanwu decotion-treated; LY, Yiqi decoction-treated; LH, Huoxue decoction-treated.

Table III. Comparison of expression levels of hypoxia-inducible factor $1 \alpha$.

\begin{tabular}{lccc}
\hline Group & Day 21 & Day 28 & Day 35 \\
\hline LB & $230.33 \pm 30.89$ & $243.29 \pm 45.10$ & $250.95 \pm 32.08^{\text {a }}$ \\
LY & $245.33 \pm 45.20$ & $243.85 \pm 31.82$ & $277.41 \pm 49.28$ \\
LH & $259.33 \pm 32.40$ & $260.00 \pm 43.14$ & $256.29 \pm 24.08^{\text {a }}$ \\
LM & $250.12 \pm 46.20$ & $269.41 \pm 34.35$ & $284.66 \pm 37.48$
\end{tabular}

${ }^{\mathrm{a}} \mathrm{P}<0.05$, compared with the LM35 group. LM, model control; LB, Buyang Huanwu decotion-treated; LY, Yiqi decoction-treated; LH, Huoxue decoction-treated.

The expression values in the LB35 and LH35 groups were $250.95 \pm 32.08$ and $256.29 \pm 24.08$, respectively. The expression levels of HIF-1 $\alpha$ were lower in the medicine-treatment groups, compared with the model control groups at the corresponding time points. However, no significant differences were found, compared with the control on days 21 and 28 post-treatment $(\mathrm{P}>0.05)$. In addition, no significant difference between the LY group and LM group were identified.

\section{Discussion}

Angiogenesis is critical for the growth, invasion and metastasis of cancer, including HCC (28). In the present study, a metastatic HCC nude mice model, which has been successfully used for HCC therapeutic agent screening, was used to investigate the effect of BYHWD and its decomposed constituents on the growth, angiogenesis and metastasis of HCC tumors. The results demonstrated that the traditional herbal medicine, BYHWD, had no significant growth inhibitory effect on HCC. However, BYHWD was found to inhibit metastasis and decrease MVD. In addition, BYHWD increased the expression of VEGF, and decreased the expression levels of RGS-5 and HIF- $1 \alpha$ on day 35 following treatment, compared with the model control.

Poon et al demonstrated that tumor MVD can be used as a predictor of recurrence following resection of HCC (29). In addition, there is increasing evidence that MVD can be considered as an indirect marker of neo-angiogenesis (30).
CD105 (endoglin) is a transforming growth factor- $\beta$ (TGF- $\beta$ ) binding protein, which is expressed on the surface of endothelial cells (31). Small and likely immature tumor blood vessels are predominantly stained by anti-CD105 monoclonal antibodies, as demonstrated previously in breast and prostate cancer $(32,33)$. Of note, several studies have shown that high MVD values, evaluated using anti-CD105 monoclonal antibodies, are significantly associated with neovascularization and prognosis in solid tumors (34). Previous data have shown that CD105 is superior to CD31 and CD34 in the evaluation of angiogenesis in certain types of cancer, as CD105 has a higher affinity for activated endothelial cells, whereas CD31 and CD34 react with normal and activated vessels (35). CD105 was identified as a significant marker of the presence and degree of neoangiogenesis. In the present study, the MVD vasculature marker was assessed by the expression of CD105. From the results, it was found that YQD or HXD treatment alone did not suppress HCC tumor growth, and no inhibition of tumor growth was induced by BYHWD until 35 days post-treatment. The present study demonstrated that the changes in tumor weight and volume in the BYHWD treatment group at 35 day were smaller than those in model group, but without statistical significance $(P>0.05)$. However, BYHWD treatment significantly reduced the MVD, indicating BYHWD treatment may affect neovascularization. In addition, the nude mice model in the present study showed $100 \%$ metastasis to the lung, indicating the HCCLM3 model in nude mice is an effective tool for investigating metastasis in HCC. However, BYHWD decreased lung metastases compared with the model groups, despite its poor inhibitory effect on tumor growth. Although increased tumor angiogenesis is usually associated with increased metastasis, it has been demonstrated that vascularization is not a marker of tumor metastasis. The present study hypothesized that the lung metastasis of HCC may have different predominant angiogenic factors or different vascular pathways, which is consistent with the results of studies by Stephens et al (36) and Zhang et al (37). Taken together, it was suggested that BYHWD had no significant inhibitory effect on HCC tumor growth, but BYHWD significantly inhibited the metastasis of HCC to the lungs and decreased tumor MVD.

Blood vessels are composed of two distinct cell types, mural cells and endothelial cells. Depending on their density, location, morphology and marker expression, the mural cells forming the outer layers of the vascular wall can be subdivided into pericytes and vascular smooth muscle cells (38). Masood et al demonstrated that the inhibition of endothelial cell mitogen VEGF signaling inhibited tumor angiogenesis and tumor cell growth, and reduced viability when expression of the VEGF was observed in the tumor cells (39). In addition, elevated levels of VEGF are reported to be associated with tumor metastasis (40). Huang et al revealed that the herbal extract, Songyou Yin, inhibited HCC invasion and inhibited the expression of VEGF in the MHCC97H HCC cells in vitro (14). However, in the present study, the expression levels of VEGF increased in the LB28 and LB35 groups, compared with the other groups, which was consistent with the earlier studies of Zhang et al (41) and Cai et al (16). Zhang et al found that BYHWD combined with bone marrow mesenchymal stem cell transplantation repaired injured blood vessels and lesion tissues via the upregulation of VEGF (41). Data has also 
revealed no survival benefit following trials of anti-VEGF monotherapy in combination with chemotherapy for the treatment of patients with metastatic cancer patients, possibly due to the complexity of tumor angiogenesis regulation (42). Thus, the present study hypothesized that BYHWD upregulated the expression of VEGF in HCC in nude mice.

By contrast, angiogenesis is driven by several other angiogenic factors, and several studies have suggested that pericytes may be important in the regulation of angiogenesis in certain tumor model systems (43). The marker profile of pericytes differs depending on the tissue of origin, whereas RGS-5 is one of the most common markers (44). RGS-5 is a signaling protein that modulates the function of $\mathrm{G}$ proteins (45). Bahrami et al found that RGS-5 was a marker of hepatic stellate cells, and its expression can mediate responses to liver injury (46). In addition, Chen et al demonstrated that the expression of RGS-5 was high in HCC (47). Furthermore, Berger et al demonstrated that RGS-5 was upregulated in pericytes during neovascularization and antitumor therapy, which reversed the tumor vasculature to an almost normal morphology and resulted in the downregulation of RGS-5 transcription (48). In the present study, whether BYHWD treatment inhibited the angiogenesis of HCC by inhibiting the expression of RGS- 5 was investigated. The results revealed that the herbal medicine treatment groups on days 28 and 35 had lower expression levels of RGS-5, compared with the LM28 and LM35 groups. Preclinical and initial clinical evidence have revealed that the normalization of abnormal vasculature is emerging as a complementary therapeutic application for the treatment of cancer (49). Therefore, in accordance with the previous studies, the present study hypothesized that BYHWD may assist in normalization of vasculature in HCC by inhibiting the expression of RGS-5.

Intratumoral hypoxia leads to the expression and stabilization of the pro-tumorigenic HIF-1 transcription factor, which is a heterodimer composed of HIF- $1 \alpha$ and HIF-1 $\beta$ (50). Kaur et al demonstrated that HIF was an essential regulatory factor in the tumor microenvironment due to its central role in promoting invasive and angiogenic properties through its upregulation of target genes containing hypoxia-responsive elements, including VEGF (50). Under hypoxic conditions, pericytes are able to secrete VEGF, and the quiescent vascular network is activated (51). Wu et al revealed that hypoxia stimulated angiogenesis to support HCC tumor growth, along with increasing the expression levels of HIF- $1 \alpha$ and VEGF (52). The survival rates of the patients with HCC exhibiting high expression levels of HIF-1 $\alpha$ were shorter, compared with those exhibiting low expression levels. In the present study, the expression levels of HIF-1 $\alpha$ in the LB35 group and LH35 group were lower, compared with that in the LM group. In accordance with a study by Fukumura et al (53), these results indicated that BYHWD treatment decreased the expression of HIF-1 $\alpha$ and contributed to the HCC tumor microenvironment.

In conclusion, the results of the present study showed that BYHWD had no significant growth inhibitory effect on HCC, however, BYHWD treatment elevated the expression level of VEGF in HCC. BYHWD may significantly inhibit the angiogenesis and metastasis of HCC in nude mice via inhibition of the expression levels of RGS-5 and HIF-1 $\alpha$, and affecting tumor vasculature structure and function. These results suggested that BYHWD may have beneficial effects on the tumor microenvironment, vasculature normalization, and inhibition of metastasis in HCC. However, further detailed experiments and investigations are required to assess the role of BYHWD therapy in the treatment of HCC.

\section{Acknowledgements}

This study was supported by the Science and Technology Foundation of Ren Ji Hospital, School of Medicine, Shanghai Jiao Tong University, Shanghai, China (grant no. 12XJ10051).

\section{References}

1. Liu M, Jiang L and Guan XY: The genetic and epigenetic alterations in human hepatocellular carcinoma: A recent update. Protein Cell 5: 673-691, 2014.

2. Jemal A, Bray F, Center MM, Ferlay J, Ward E and Forman D: Global cancer statistics. CA Cancer J Clin 61: 69-90, 2011.

3. Bruix J, Gores GJ and Mazzaferro V: Hepatocellular carcinoma: Clinical frontiers and perspectives. Gut 63: 844-855, 2014.

4. Carmeliet $P$ and Jain RK: Molecular mechanisms and clinical applications of angiogenesis. Nature 473: 298-307, 2011.

5. Pang R and Poon RT: Angiogenesis and antiangiogenic therapy in hepatocellular carcinoma. Cancer Lett 242: 151-167, 2006.

6. Folkman J: Fundamental concepts of the angiogenic process. Curr Mol Med 3: 643-651, 2003.

7. Zhao J, Lin W, Cao Z, Zhuang Q, Zheng L, Peng J and Hong Z: Total alkaloids of Rubus alceifolius Poir inhibit tumor angiogenesis through suppression of the Notch signaling pathway in a mouse model of hepatocellular carcinoma. Mol Med Rep 11: 357-361, 2015.

8. Casazza A, Laoui D, Wenes M, Rizzolio S, Bassani N, Mambretti M, Deschoemaeker S, Van Ginderachter JA, Tamagnone $\mathrm{L}$ and Mazzone $\mathrm{M}$ : Impeding macrophage entry into hypoxic tumor areas by Sema3A/Nrp1 signaling blockade inhibits angiogenesis and restores antitumor immunity. Cancer Cell 24: 695-709, 2013.

9. Lee E, Koskimaki JE, Pandey NB and Popel AS: Inhibition of lymphangiogenesis and angiogenesis in breast tumor xenografts and lymph nodes by a peptide derived from transmembrane protein 45A. Neoplasia 15: 112-124, 2013.

10. Konkimalla VB and Efferth T: Evidence-based Chinese medicine for cancer therapy. J Ethnopharmacol 116: 207-210, 2008.

11. Hu Y, Wang S, Wu X, Zhang J, Chen R, Chen M and Wang Y: Chinese herbal medicine-derived compounds for cancer therapy: A focus on hepatocellular carcinoma. J Ethnopharmacol 149: 601-612, 2013.

12. Han SY and Li PP: Progress of research in antitumor mechanisms with Chinese medicine. Chin J Integr Med 15: 316-320, 2009.

13. Dong Y, Lu B, Zhang X, Zhang J, Lai L, Li D, Wu Y, Song Y, Luo J, Pang X, et al: Cucurbitacin E, a tetracyclic triterpenes compound from Chinese medicine, inhibits tumor angiogenesis through VEGFR2-mediated Jak2-STAT3 signaling pathway. Carcinogenesis 31: 2097-2104, 2010.

14. Huang XY, Wang L, Huang ZL, Zheng Q, Li QS and Tang ZY: Herbal extract 'Songyou Yin' inhibits tumor growth and prolongs survival in nude mice bearing human hepatocellular carcinoma xenograft with high metastatic potential. J Cancer Res Clin Oncol 135: 1245-1255, 2009.

15. Zhao LD, Wang JH, Jin GR, Zhao Y and Zhang HJ: Neuroprotective effect of Buyang Huanwu decoction against focal cerebral ischemia/reperfusion injury in rats-time window and mechanism. J Ethnopharmacol 140: 339-344, 2012.

16. Cai G, Liu B, Liu W, Tan X, Rong J, Chen X, Tong L and Shen J: Buyang Huanwu decoction can improve recovery of neurological function, reduce infarction volume, stimulate neural proliferation and modulate VEGF and Flk1 expressions in transient focal cerebral ischaemic rat brains. J Ethnopharmacol 113: 292-299, 2007.

17. Jain RK: Normalization of tumor vasculature: An emerging concept in antiangiogenic therapy. Science 307: 58-62, 2005. 
18. Shang B, Cao Z and Zhou Q: Progress in tumor vascular normalization for anticancer therapy: Challenges and perspectives. Front Med 6: 67-78, 2012.

19. Tang ZY, Ye SL, Liu YK, Qin LX, Sun HC, Ye QH, Wang L, Zhou J, Qiu SJ, Li Y, et al: A decade's studies on metastasis of hepatocellular carcinoma. J Cancer Res Clin Oncol 130: 187-196, 2004

20. Xu JQ (ed): Formulas of Chinese Medicine. Shanghai Scientific \& Technical Publishers, Shanghai, pp1-298, 1985.

21. Chinese Pharmacopoeia Commission. Chinese pharmacopoeia. Chemical Industry Press, Beijing China pp547, 2005.

22. Kang M, Ou H, Wang R, Liu W, Mao Y and Tang A: Effect of trichosanthin on apoptosis and telomerase activity of nasopharyngeal carcinomas in nude mice. J BUON 18: 675-682, 2013.

23. Institute of Laboratory Animal Resources (US). Committee on Care, Use of Laboratory Animals, and National Institutes of Health (US). Division of Research Resources: Guide for the care and use of laboratory animals. 1st edition. National Academies Press, Washington, DC, 1996.

24. Li Y, Tang Z, Ye L, Liu B, Liu K, Chen J and Xue Q: Establishment of a hepatocellular carcinoma cell line with unique metastatic characteristics through in vivo selection and screening for metastasis-related genes through cDNA microarray. J Cancer Res Clin Oncol 129: 43-51, 2003.

25. Zhang T, Sun HC, Xu Y, Zhang KZ, Wang L, Qin LX, Wu WZ, Liu YK, Ye SL and Tang ZY: Overexpression of platelet-derived growth factor receptor alpha in endothelial cells of hepatocellular carcinoma associated with high metastatic potential. Clin Cancer Res 11: 8557-8563, 2005.

26. Ye QH, Qin LX, Forgues M, He P, Kim JW, Peng AC, Simon R, Li Y, Robles AI, Chen Y, et al: Predicting hepatitis B virus-positive metastatic hepatocellular carcinomas using gene expression profiling and supervised machine learning. Nat Med 9: 416-423, 2003.

27. Weidner N, Semple JP, Welch WR and Folkman J: Tumor angiogenesis and metastasis-correlation in invasive breast carcinoma N Engl J Med 324: 1-8, 1991.

28. Zhang ZL, Liu ZS and Sun Q: Effects of thalidomide on angiogenesis and tumor growth and metastasis of human hepatocellular carcinoma in nude mice. World J Gastroenterol 11: 216-220, 2005.

29. Poon RT, Ng IO, Lau C, Yu WC, Yang ZF, Fan ST and Wong J: Tumor microvessel density as a predictor of recurrence after resection of hepatocellular carcinoma: A prospective study. J Clin Oncol 20: 1775-1785, 2002.

30. Ng IO, Poon RT, Lee JM, Fan ST, Ng M and Tso WK: Microvessel density, vascular endothelial growth factor and its receptors Flt-1 and Flk-1/KDR in hepatocellular carcinoma. Am J Clin Pathol 116: 838-845, 2001.

31. Yao Y, Kubota T, Takeuchi H and Sato K: Prognostic significance of microvessel density determined by an anti-CD105/endoglin monoclonal antibody in astrocytic tumors: Comparison with an anti-CD31 monoclonal antibody. Neuropathology 25: 201-206, 2005.

32. Wikström P, Lissbrant IF, Stattin P, Egevad L and Bergh A: Endoglin (CD105) is expressed on immature blood vessels and is a marker for survival in prostate cancer. Prostate 51: 268-275, 2002.

33. Fonsatti $\mathrm{E}$ and Maio M: Highlights on endoglin (CD105): From basic findings towards clinical applications in human cancer. J Transl Med 2: 18, 2004

34. Imura $S$, Miyake $H$, Izumi $K$, Tashiro $S$ and Uehara $H$ : Correlation of vascular endothelial cell proliferation with microvesse density and expression of vascular endothelial growth factor and basic fibroblast growth factor in hepatocellular carcinoma. J Med Invest 51: 202-209, 2004

35. Tanaka F, Otake Y, Yanagihara K, Kawano Y, Miyahara R, Li M, Yamada T, Hanaoka N, Inui K and Wada H: Evaluation of angiogenesis in non-small cell lung cancer comparison between anti-CD34 antibody and anti-CD105 antibody. Clin Cancer Res 7: 3410-3415, 2001.
36. Stephens TD, Bunde CJ and Fillmore BJ: Mechanism of action in thalidomide teratogenesis. Biochem Pharmacol 59: 1489-1499, 2000.

37. Zhang ZL, Liu ZS and Sun Q: Anti-tumor effect of thalidomide and paclitaxel on hepatocellular carcinoma in nude mice. Chin Med J (Engl) 118: 1688-1694, 2005.

38. Zhou L, Sohet F and Daneman R: Purification and culture of central nervous system pericytes. Cold Spring Harb Protoc 2014: $581-583,2014$.

39. Masood R, Cai J, Zheng T, Smith DL, Hinton DR and Gill PS: Vascular endothelial growth factor (VEGF) is an autocrine growth factor for VEGF receptor-positive human tumors. Blood 98: 1904-1913, 2001.

40. Hirakawa S, Brown LF, Kodama S, Paavonen K, Alitalo K and Detmar M: VEGF-C-induced lymphangiogenesis in sentinel lymph nodes promotes tumor metastasis to distant sites. Blood 109: 1010-1017, 2007.

41. Zhang YK, Han XY and Che ZY: Effects of buyang huanwu tang combined with bone marrow mesenchymal stem cell transplantation on the expression of VEGF and $\mathrm{Ki}-67$ in the brain tissue of the cerebral ischemia-reperfusion model rat. J Tradit Chin Med 30: 278-282, 2010.

42. Quesada AR, Medina MA and Alba E: Playing only one instrument may be not enough: Limitations and future of the antiangiogenic treatment of cancer. Bioessays 29: 1159-1168, 2007.

43. Shaheen RM, Tseng WW, Davis DW, Liu W, Reinmuth N, Vellagas R, Wieczorek AA, Ogura Y, McConkey DJ, Drazan KE, et al: Tyrosine kinase inhibition of multiple angiogenic growth factor receptors improves survival in mice bearing colon cancer liver metastases by inhibition of endothelial cell survival mechanisms. Cancer Res 61: 1464-1468, 2001.

44. Bondjers C, Kalén M, Hellström M, Scheidl SJ, Abramsson A, Renner O, Lindahl P, Cho H, Kehrl J and Betsholtz C: Transcription profiling of PDGF-B deficient embryos identifies RGS5 as a novel marker for pericytes and vascular smooth muscle cells. Am J Pathol 162: 721-729, 2003.

45. Zhou J, Moroi K, Nishiyama M, Usui H, Seki N, Ishida J, Fukamizu A and Kimura S: Characterization of RGS5 in regulation of $\mathrm{G}$ protein-coupled receptor signaling. Life Sci 68: 1457-1469, 2001.

46. Bahrami AJ, Gunaje JJ, Hayes BJ, Riehle KJ, Kenerson HL, Yeung RS, Stempien-Otero AS, Campbell JS and Mahoney WM Jr: Regulator of G-protein signaling-5 is a marker of hepatic stellate cells and expression mediates response to liver injury. PLoS One 9: e108505, 2014.

47. Chen X, Higgins J, Cheung ST, Li R, Mason V, Montgomery K, Fan ST, van de Rijn M and So S: Novel endothelial cell markers in hepatocellular carcinoma. Mod Pathol 17: 1198-1210, 2004.

48. Berger M, Bergers G, Arnold B, Hämmerling GJ and Ganss R: Regulator of G-protein signaling-5 induction in pericytes coincides with active vessel remodeling during neovascularization. Blood 105: 1094-1101, 2005.

49. Carmeliet P and Jain RK: Principles and mechanisms of vessel normalization for cancer and other angiogenic diseases. Nat Rev Drug Discov 10: 417-427, 2011.

50. Kaur B, Khwaja FW, Severson EA, Matheny SL, Brat DJ and Van Meir EG: Hypoxia and the hypoxia-inducible-factor pathway in glioma growth and angiogenesis. Neuro Oncol 7: 134-153, 2005.

51. Qing G and Simon MC: Hypoxia inducible factor-2alpha: A critical mediator of aggressive tumor phenotypes. Curr Opin Genet Dev 19: 60-66, 2009

52. Wu XZ, Xie GR and Chen D: Hypoxia and hepatocellular carcinoma: The therapeutic target for hepatocellular carcinoma. J Gastroen Hepatol 22: 1178-1182, 2007.

53. Fukumura D and Jain RK: Tumor microvasculature and microenvironment: Targets for anti-angiogenesis and normalization. Microvasc Res 74: 72-84, 2007. 